\title{
Téoros
}

Revue de recherche en tourisme

\section{Les publications touristiques spécialisées : trop souvent au diapason de leurs annonceurs}

\section{Paul Simier}

Volume 4, numéro 1, mars 1985

L’information touristique

URI : https://id.erudit.org/iderudit/1080741ar

DOI : https://doi.org/10.7202/1080741ar

Aller au sommaire du numéro

Éditeur(s)

Université du Québec à Montréal

ISSN

0712-8657 (imprimé)

1923-2705 (numérique)

Découvrir la revue

Citer cet article

Simier, P. (1985). Les publications touristiques spécialisées : trop souvent au diapason de leurs annonceurs. Téoros, 4(1), 18-19.

https://doi.org/10.7202/1080741ar d'utilisation que vous pouvez consulter en ligne.

https://apropos.erudit.org/fr/usagers/politique-dutilisation/ 


\title{
Les publications touristiques spécialisées: trop souvent au diapason de leurs annonceurs
}

\author{
par Paul Simier*
}

Elles sont discrètes parce que leur diffusion se limite au cercle restreint du monde des professionnels du voyage. Pourtant, elles se sont mutipliées ces dernières années, à croire que le marché du voyage est florissant. Les publications touristiques spécialisées, au-delà de ces caractéristiques, ont souvent en commun d'être d'abord au diapason de leurs commanditaires.

D'aucuns diront qu'il est encore heureux que nous ayons ainsi une presse écrite spécialisée au Québec. La presse en français destinée aux professionnels du voyage r'a pas encore dix ans. Les seuls imprimés venaient auparavant de Toronto et de NewYork.

Marketing Voyages, la première publication en français à paraitre il y a huit ans. avait sans doute le tort d'être mensuelle. Cela explique la création peu de temps plus tard d'un hebdomadaire, Le Journal des Voyages, qui venait exploiter le créneau de la nouvelle que ne couvrait pas exactement le magazine.

La concurrence qui s'établissait ainsi explique aussi le lancement un peu plus tard par l'éditeur de Marketing Voyages d'un autre hebdomadaire, Bulletin Voyages.

Tourisme Plus a vu le jour il y a cinq ans. Avec ce mensuel qui très vite allait se convertir en bi-mensuel, I'échiquier était complété. Toutes ces créations ont eu lieu en seulement trois ans.

II y a quelques mois, Bulletin Voyages d'abord, puis Le Journal des Voyages lançaient une deuxième édition hebdoma daire.

\section{Des changements qui relèvent de I'instabilité}

Ainsi résumé, l'histoire de la presse écrite québécoise spécialisée fait fi de tous les heurts et de toutes les douleurs qui ont marqué la vie de ces jeunes publications. Au-delà de la petite histoire, ces aléas sont importants, car ils permettent de comprendre et les problèmes de survie mème de ces publications et la dimension de l'information qu'elles transmettent.

"Paul Simier est chroniqueur en tourisme.

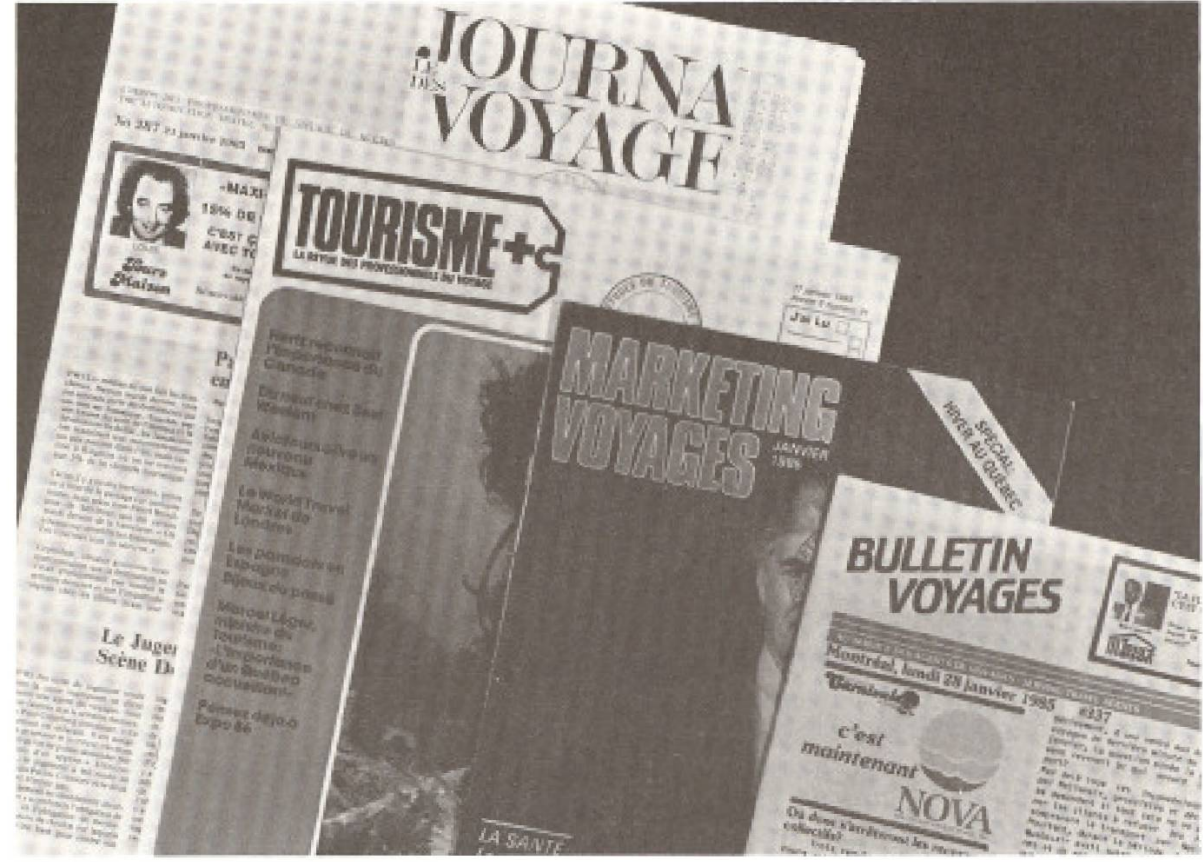

Les publications spécialisées: d'abord des outils pour faciliter le travail des professionnels.

Marketing Voyages en est à présent à son troisiẻme éditeur propriétaire. Bulletin Voyages à son quatrieme. Le Journal des Voyages à son deuxième. Seul Tourisme Plus conserve à la base la me̊me actionnaire principale qu'il avait au départ.

Ces changements successifs qui n'ont épargné aucune publication s'expliquent par l'instabilité financière qui les a toutes affectées un jour ou l'autre.

Le marché de l'information spécialisée n'est donc pas aussi florissant qu'on pourrait l'imaginer. Distribuées gratuitement selon sensiblement les mèmes listes - soit entre 5000 et 6000 destinataires - toutes les publications ont en commun de dépendre presque exclusivement de leurs ressources publicitaires. Les abonnements r'ont qu'un intérêt symbolique dans leurs revenus.

Les campagnes de promotion des abonnements auxquelles s'adonnent épisodiquement l'une ou l'autre visent plus à prouver leur sérieux aux organismes de contrôle de la circulation pour rassurer les annonceurs.
Les publications spécialisées ont en commun de dépendre presque exclusivement de leurs ressources publicitaires.

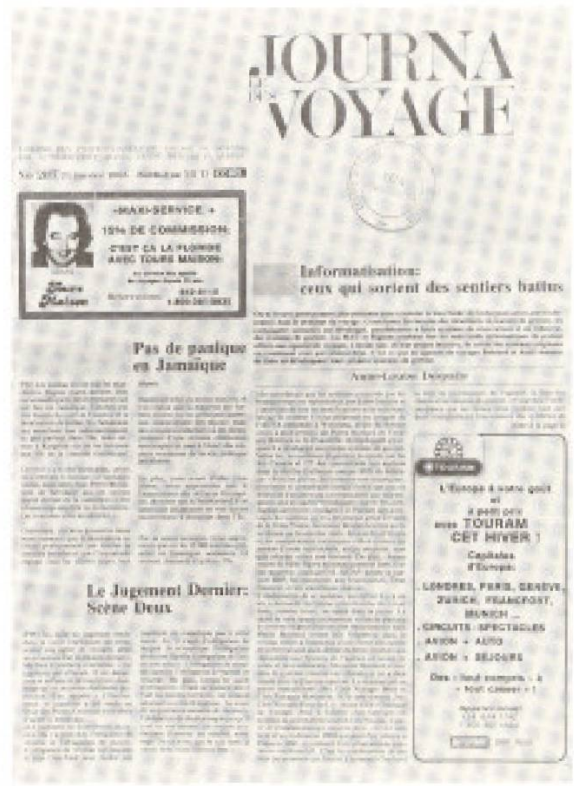




\section{De faibles moyens rédactionnels}

A un rythme encore plus rapide que celui auquel les titres ont changé de propriétaire ou d'actionnaires, on assiste à une valse constante des collaborateurs. Un autre point commun entre toutes les publications: rares sont les rédacteurs d'expérience qui collaborent à ces revues. Si cela se produit, la situation est très éphémère.

L'explication est encore d'ordre financier. Plusieurs publications rémunèrent encore leurs collaborateurs sous la forme de vovages, c'est-à-dire envoient en tournées dites de familiarisation, organisées par des transporteurs, des offices de tourisme ou des voyagistes, des collaborateurs pour qui c'est â la fois un salaire et une récompense.

Imaginez de jeunes journalistes ou des personnes aspirant à le devenir ainsi exploites. Constat général: tout cela ne paie pas le loyer. De plus, ce genre de lien n'incite pas l'auteur d'un texte à être détaché des inté:rêts de ceux-là même qui lancent les invitations et qui sont aussi les annonceurs.

Cela a toujours entraîné une rotation rapide des collaborateurs. Seul Le Journal des Voyages paie ses collaborateurs depuis déjà quelques temps. Les autres publications n'emploient pas vraiment de permanents.

\section{Penser publicité ou information}

On ne doit pas attendre d'une publication spécialisée qu'elle nous décrive les beautes des cocotiers, le ciel bleu et les charmes des folles nuits. Destinees d'abord aux intermédiaires que sont les conseillers en voyages, aux grossistes et aux divers fournisseurs de services touristiques, ces publications doivent d'abord donner des outils à tous ces professionnels pour travailler.

Les projets de développement, les stratégies de mise en marche, les caractéristiques des nouveaux produits, les nouveaux systemes d'organisation des entreprises, les actions menées par les organisations professionnelles doivent figurer parmi les priorités de toute personne qui fait carrière dans cette industrie.

La bonne publication devrait de la même façon couvrir tous les domaines de l'information, en touchant aussi bien à tous les secteurs concernés par le tourisme: transport, hôtellerie, actions réceptives, assurances, devises, information, mise en marché, marketing, formation, etc...

Trop souvent on doit faire le constat que I'information se trouve branchée en ligne directe sur les commanditaires. On peut lire ici un article et trouver présentée en parallèle une publicité de la compagnie concernée. Si encore on faisait dire des choses intéressantes aux interviews... Souvent aussi le contenu de l'entrevue n'est qu'une pâle redite de ces mémes réclames.

A croire qu'ils pensent d'abord à faire plaisir à leurs annonceurs plutốt qu'à informer le plus complètement possible leurs lecteurs. Il est vrai qu'il subsiste des situations de conflits d'intéréts, considérées comme normales, dans le milieu. L'éditeur d'une publication n'est-il pas en meme temps chargé pour le Québec du compte publicitaire d'un office de tourisme étranger et responsable à l'occasion d'inviter des journalistes à visiter ce pays?

\section{Une amélioration}

Avec des journalistes débutants mal payés ou pas payés du tout si ce n'est en laissez= passer et en invitations, avec des éditeurs soucieux de s'en sortir et de faire de l'argent en sacrifiant souvent l'information aux intérèts de leurs bailleurs de fonds, il ne faut donc pas s'attendre à toujours trouver des publications sérieuses.

Parce que longtemps on les a confondues, parce que le taux de lecture effectif fait de plus en plus la difference, des changements apparaissent. Certains éditeurs ont investi (un peu) pour tenter d'apporter une information plus solide, axée sur les besoins de leurs lecteurs.

De plus en plus aussi les annonceurs évaluent le sérieux de leurs placements et font faire des enquêtes d'impact des titres. Et comme cet argument est décisif pour les éditeurs concernés, les changements se multiplient.
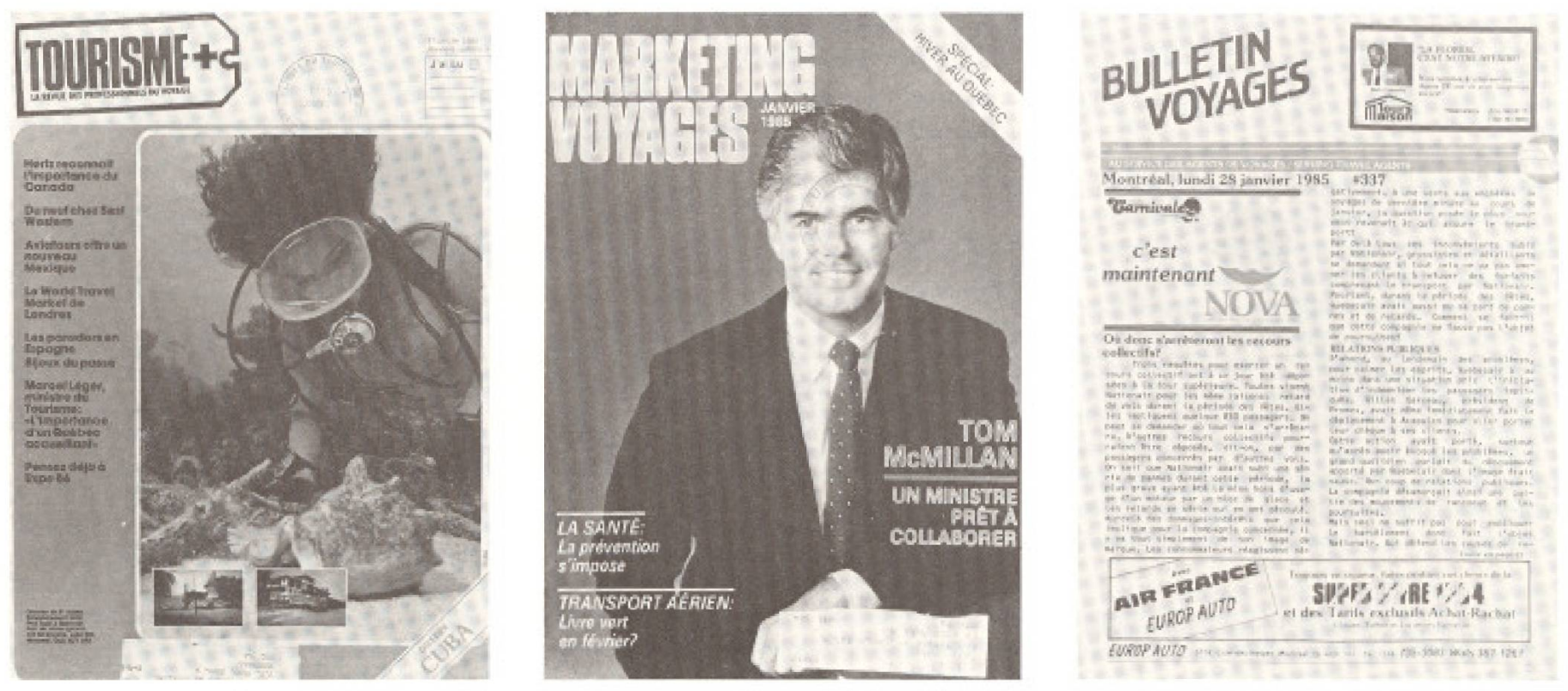